\title{
Avaliação antimicrobiana e moduladora do extrato de Achyrocline satureioides (Lam.) DC. frente a Escherichia coli
}

\author{
Hadassa Moura Pinheiro ${ }^{\mathrm{a}}$, Irineu Ferreira da Silva Neto ${ }^{\mathrm{a}^{*}} \oplus$, Fabiana Alvez Barros ${ }^{\mathrm{a}}{ }^{\oplus}$, \\ Nadghia Figueiredo Leite Sampaio ${ }^{\mathrm{a}} \bullet$, Cícera Natalia Figueiredo Leite Gondim ${ }^{\mathrm{b}}$ \\ a Faculdade de Medicina Estácio de Juazeiro do Norte, Brasil \\ b Universidade Regional do Cariri, Brasil \\ *Autor correspondente (yrineuferreira@gmail.com)
}

\section{N F O}

\section{Keyworks}

ethanol extract

modulation

antimicrobial potential
Palavras-chaves extrato etanólico modulação potencial antimicrobiano

\begin{abstract}
A B S T R A C T
Antimicrobial and modulatory evaluation of Achyrocline satureioides (Lam.) DC extract. against Escherichia coli

Escherichia coli is a Gram-negative bacterium present in gastrointestinal disorders and is responsible for causing several symptoms, being diarrhea or more common, due to the production of enterotoxins. Note that E. coli strains are becoming resistant due to the use of antimicrobials. Through the search for therapies, medicinal plants have been a source of new research and studies, enabling technical methods in practice. Thus, the objective was to evaluate the antimicrobial and modulatory effect of the ethanol extract of the medicinal plant Achyrocline satureioides against E. coli serotypes. The inflorescences of $A$. satureioides were collected in March 2019 and, after obtaining the extract, the Minimum Inhibitory Concentration (MIC) assay was performed, and its antibiotic and synergistic activity was evaluated by microdilution. After analyzing the data, it was found that the extract in its form obtained a result of CMI $\geq 1024 \mu \mathrm{g} / \mathrm{mL}$. A synergistic effect of the extracts was observed when combined with amikacin in the EPEC and ETEC serotypes, as well as with gentamicin in the ETEC serotype. In the other analyzes there was no synergistic or antagonistic effect. The ethanol extract of $A$. satureioides inflorescences in association with aminoglycosides showed a synergistic effect as tested lines, proving a tendency to the antimicrobial effect, therefore, it is shown that an association of the tested antibiotics increased the antimicrobial potential. Further research will be important for further certification that this is an alternative method in the treatment of gastrointestinal diseases.
\end{abstract}

\section{R E S U M O}

A Escherichia coli é uma bactéria Gram-negativa presente em desordens gastrointestinais e é responsável por causar diversos sintomas, sendo a diarreia o mais corrente, devido a produção de enterotoxinas. Observa-se que as cepas de $E$. coli estão se tornando resistentes devido ao uso inadequado de antimicrobianos. Mediante a busca de terapias, as plantas medicinais têm sido fonte de novas pesquisas e estudos, possibilitando eficazes métodos aplicados na prática clínica. Dessa forma, objetivou-se avaliar o efeito antimicrobiano e modulador do extrato etanólico da planta medicinal Achyrocline satureioides frente a sorotipos de E. coli. As inflorescências de A. satureioides foram coletadas no mês de março de 2019 e, após a obtenção do extrato, foi realizado o ensaio de Concentração Inibitória Mínima (CIM), bem como foi avaliado a sua atividade antibiótica e sinérgica por meio de microdiluição. Após a análise dos dados constatou-se que o extrato na sua forma isolada obteve resultado de CMI $\geq 1024 \mu \mathrm{g} / \mathrm{mL}$. Foi observado um efeito sinérgico dos extratos quando combinados com amicacina nos sorotipos EPEC e ETEC, como também na gentamicina no sorotipo ETEC. Nas demais análises não houve efeito sinérgico e nem antagônico. $\mathrm{O}$ extrato etanólico das inflorescências da $A$. satureioides em associação com os aminoglicosídeos mostrou efeito sinérgico frente as linhagens testadas, apresentando-se uma tendência ao efeito antimicrobiano, portanto, demostra-se que a associação dos antibióticos testados aumentou o potencial antimicrobiano. Pesquisas posteriores serão importantes para maior certificação de que essa seja um método alternativo no tratamento de infecções gastrointestinais. 


\section{INTRODUÇÃO}

Doenças transmitidas por alimentos são problemas presentes enfrentados pela saúde pública desde o início da civilização. Em 2017, no Brasil, foram identificados 133 surtos e 2014 doentes (Brasil, 2017). Escherichia coli, diarreiogênicas, rotavirus, Salmonella spp., Shigella spp., Campylobacter jejuni e Campylobacter coli, cryptosporidium spp., Entamoeba histolytica e Giardia lamblia são os agentes etiológicos e epidemiológicos de maior relevância clínica responsáveis por causar estas patologias (Gomes et al., 2017), tendo em vista que $90,5 \%$ dos surtos são causados pelas bactérias e estas, por sua vez, são encontradas recorrentemente em esgotos (Brasil, 2017).

Os principais sintomas causados por esses agentes são: diarreia, vômito, dor abdominal e náuseas (Brasil, 2017). A diarreia é o sintoma mais perigoso que afeta crianças e adultos na infecção alimentar e tem como principal patógeno causador a E. coli (Farfán García et al., 2016).

E. coli são bacilos Gram-negativos, anaeróbios facultativos que pertencem a família Enterobacteriaceae e recebeu o nome do pediatra alemão Theodor Escherich (Gomes et al., 2016). Foram descritos seis patotipos de E. coli em doenças diarreicas: E. coli enteropatogênica, E. coli enterotoxigênica, E. coli enteroinvasora, E. coli enterohemorrágica ou $E$. coli produtora da toxina de Shiga, E. coli enteroagregativa e $E$. coli aderente difusa (Souza et al., 2016).

$\mathrm{O}$ tratamento para diarreia causada por vírus, faz-se através de reposição de líquidos e cuidados com a alimentação, entretanto o tratamento do agente bacteriano faz-se com o antibiótico apropriado. Dessa forma, é necessário controlar vigorosamente a conduta clínica caso a caso, uma vez que estudos abordam o surgimento de cepas da família Enterobacteriaceae, resistentes aos agentes antimicrobianos (Zhou et al., 2018) os quais desenvolveram formas multirresistentes (Moresco et al., 2017).

Pesquisas indicam que existam fontes potencias de novas moléculas antimicrobianas em plantas medicinais (Anyanwu e Okoye, 2017). Tem-se várias plantas sendo utilizadas para diversos fins terapêuticos, como também, existem produtos naturais servindo como percussores de moléculas bioativas para a síntese de novos antibióticos (Guimarães et al., 2010).

A Achyrocline satureioides, conhecida popularmente como "marcela" ou "macela" é uma planta bastante utilizada por apresentar diversas propriedades farmacológicas. Estudos in vivo e in vitro confirmaram a sua efetividade antiinflamatória, hepatoprotetora, antioxidante, imunomoduladora, antitumoral, fotoprotetora e com destaque para a propriedade antimicrobiana (Salgueiro et al., 2016).

Retta et al. (2012) destaca que a planta medicinal A. satureioides é uma erva aromática anual, tamanho médio e nativa da América, se estende por todo o continente assim como na Europa e África (Salgueiro et al., 2016). Sabini et al. (2016) relata que está pertence à família Asteraceae, sendo amplamente consumida por infusão, decocção, maceração e como xarope.

Devido a sua grande utilização pela população, por meio do conhecimento empírico no combate a desordens gastrointestinais, desperta-se interesse em pesquisas e testes que comprovem a eficácia terapêutica da respectiva planta medicinal, contribuindo assim para indústria farmacêutica na descoberta de novos fármacos (Cariddi et al., 2015).

Dessa forma, objetivou-se avaliar o efeito antimicrobiano e modulador do extrato etanólico da planta medicinal $A$. satureioides frente a sorotipos de E. coli.

\section{MATERIAL E MÉTODOS}

\section{Material vegetal}

As inflorescências de $A$. satureioides, foram coletadas no mês de março de 2019 no sítio Barreiros, de Latitude: $07^{\circ} 18^{\prime} 48^{\prime \prime}$ S Longitude: $38^{\circ}$ $566^{\prime} 44^{\prime \prime} \mathrm{W}$, na cidade de Milagres - Ceará, Brasil. O material foi depositado no Herbário Caririense Dárdano de Andrade Lima - LMBM da Universidade Regional do Cariri - URCA e encontra-se em fase de identificação.

\section{Microrganismos}

Os sorotipos de E. coli foram fornecidos pelo Laboratório de Microbiologia e Biologia Molecular da Universidade Regional do Cariri (URCA). Utilizou-se os sorotipos $E$. coli enteropatogênica (EPEC) e a E. coli enterotoxigênica (ETEC). Estas, por sua vez, foram inoculadas no meio de cultura Brain Heart Infusion Agar- BHI (Sigma ${ }^{\circledR}$ ) e armazenadas sob refrigeração a $5^{\circ} \mathrm{C}$, durante $72 \mathrm{~h}$.

\section{Meios de cultura}

Incialmente, preparou-se o BHI líquido a $10 \%$. Pesou-se 20g de BHI, adicionando-se posteriormente $200 \mathrm{~mL}$ de água destilada, e em seguida, foi homogeneizado e identificado.

\section{Preparação do extrato}

Para a preparação do extrato os frutos foram retirados da planta e pesados resultando em $56,93 \mathrm{~g}$ e, posteriormente, foram submersos em $650 \mathrm{~mL}$ de álcool PA. Colocou-se em um pote de vidro e 
cobriu-se em um tempo estimado de 72h. Após esse período, retirou-se as flores da marcela do álcool, filtrou-se à vácuo em um kitassato com ajuda de um funil de Büchner, resultando no extrato alcoólico 393,04 mL. O extrato foi colocado no evaporador rotativo em um tempo de 3 horas e depois colocouse no banho maria para secar, permanecendo por 7 dias e, ao final, formou-se uma pasta de extrato de A. satureioides na quantidade de $1,956 \mathrm{~g}$.

\section{Preparação do inóculo}

As cepas foram inoculadas em BHI ágar e mantidas na estufa a $35^{\circ} \mathrm{C}$ por $24 \mathrm{~h}$. Uma alçada do inóculo foi inserida em tubos de ensaio contendo solução salina. Foi observado a turvação comparando com a escala de McFarland para obter uma concentração de $10^{5}$ UFC.mL mb $^{-1}$ (Unidade Formadora de Colónias) das bactérias. Os inóculos foram preparados em triplicata para garantir maior segurança nos resultados obtidos.

\section{Concentração Inibitória Mínima (CIM)}

A Concentração Inibitória Mínima (CIM) é definida como a menor concentração capaz de impedir o crescimento bacteriano, nas cavidades da placa de microdiluição conforme detectado macroscopicamente (NCCLS, 2003).

A CIM foi realizada pelo método de microdiluição em caldo. $\mathrm{O}$ inóculo foi depositado em solução salina para formação de uma suspensão de $10^{5}$ UFC. $\mathrm{mL}^{-1}$, as concentrações das substâncias variaram de 1024 a $8 \mu \mathrm{g} \cdot \mathrm{mL}^{-1}$.

Em seguida foi distribuído $100 \mu \mathrm{L}$ desta solução em cada cavidade da placa de microdiluição e logo após adicionou-se $100 \mu \mathrm{L}$ de extrato na primeira cavidade, passando para as demais, através de sucessivas diluições na proporção de $1: 1$, até a penúltima cavidade. A última cavidade foi reservada para controle. A placa foi colocada na estufa numa temperatura de aproximadamente $37^{\circ} \mathrm{C}$, por um período de 24 horas. As placas contendo bactérias reveladas com corante específico, a resazurina, um indicador colorimétrico de óxido-redução. Para realizar a leitura do teste fez-se necessário adicionar $20 \mu \mathrm{L}$ da solução em cada cavidade das placas e incubá-las por $1 \mathrm{~h}$ em temperatura ambiente (Salvat et al., 2001).
A revelação da CIM considerada como inibição do crescimento para os poços que permaneceram com a coloração azul e não-inibição os que obtiveram coloração vermelha.

\section{Modulação da atividade antibiótica por microdiluição}

As substâncias foram testadas em concentração sub-inibitória (MIC/8). Foi distribuídos em eppendorfs (para modulação) uma solução contendo BHI, inóculo e a substância isolada (MIC/8). Para a coluna controle (do antibiótico) eppendorfs foram preenchidos com BHI e inóculo. As soluções dos eppendorfs foram distribuídas em colunas onde em cada poço recebeu $100 \mu \mathrm{L}$ da solução. Toda a distribuição foi feita no sentido alfabético da placa. Em seguida, $100 \mu \mathrm{L}$ do antibiótico na concentração de $1024 \mu \mathrm{g} \cdot \mathrm{mL}^{-1}$ foram misturados ao primeiro poço, procedendo-se a microdiluição em série, numa proporção de $1: 1$ até a penúltima cavidade. As concentrações de antimicrobianos variaram gradualmente de 1024 a $2,44 \mu \mathrm{g} \cdot \mathrm{mL}^{-1}$.

\section{Análise estatística}

Os resultados foram feitos em triplicata e expressos como média das repetições. Para análise estatística do teste de modulação com os antibióticos e construção dos gráficos foram aplicados à análise de variância Two-way ANOVA seguido pelos pós teste de Bonferroni utilizando o software GraPhadPrism 5.0. Foram considerados relevantes valores com o $\mathrm{p}<0,0001$.

\section{RESULTADOS E DISCUSSÃO}

A CIM do extrato de A. satureioides para E. coli enteropatogênica (EP) e $E$. coli enterotoxigênica (ET) foram $\geq 1024 \mu \mathrm{g} \cdot \mathrm{mL}^{-1}$. Assim, o extrato não apresentou atividade antimicrobiana, uma vez que concentrações superiores ao valor supracitado são clinicamente irrelevantes (Seelig e Grazziotin, 2014).

Com base nos valores da CIM, foi avaliado a associação do extrato com drogas antimicrobianas da classe dos aminoglicosídeos e das lincosamidas, pelo método de microdiluição (Figuras 1 e 2). 


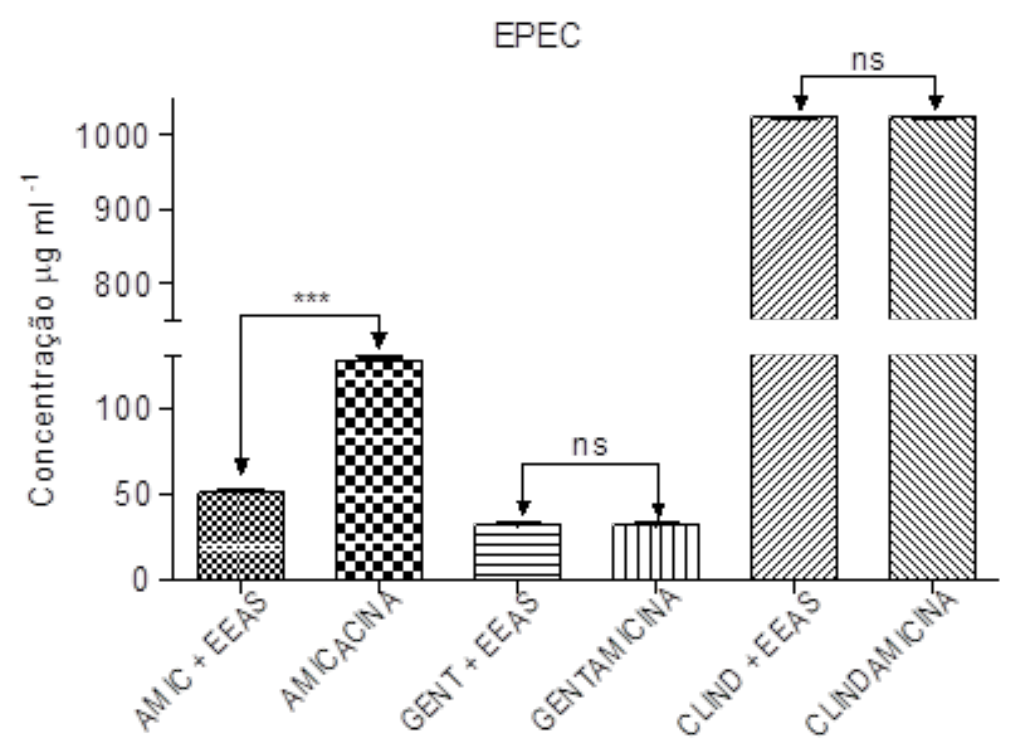

EPEC: E. coli enteropatogênica; EEAS: Extrato etanólico de A. satureioides.; Amic: Amicacina; Gent.: Gentamicina; Clind.: Clindamicina. $* * *$ Valor estatisticamente significante com $\mathrm{p}<0.0001$. ns: não significante estatisticamente.

Figura 1 - Atividade moduladora de antibióticos do extrato da A. satureioides contra E. coli enteropatogênica - EP.

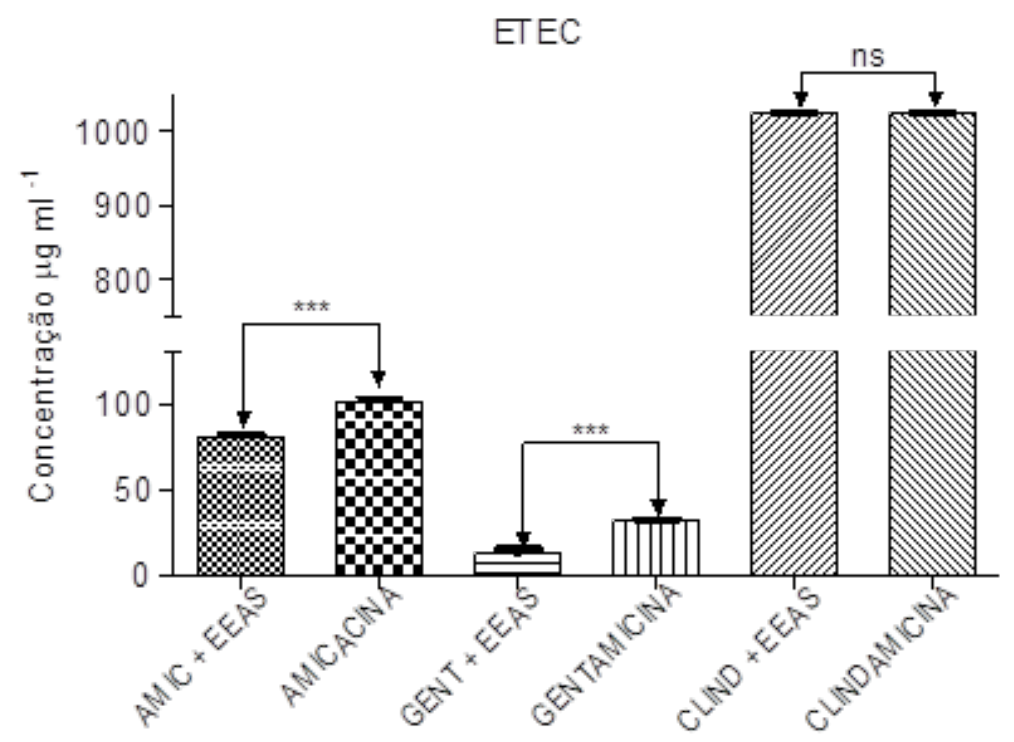

ETEC: E. coli enterotoxigênica; EEAS: Extrato etanólico de A. satureioides.; Amic: Amicacina; Gent.: Gentamicina; Clind.: Clindamicina. *** Valor estatisticamente significante com $\mathrm{p}<0.0001$. ns: não significante estatisticamente.

Figura 2 - Atividade moduladora de antibióticos do extrato da A. satureioides contra E. coli enterotoxigênica $-\mathrm{ET}$.

A amicacina obteve resultado significativo em associação com o extrato no combate a EPEC e ETEC, podendo evidenciar efeito sinérgico no estudo, uma vez que, o seu uso isolado necessitou-se uma concentração de $128 \mu \mathrm{g} \cdot \mathrm{mL}^{-1}$ na EPEC e $101,59 \mu \mathrm{g} \cdot \mathrm{mL}^{-1}$ na ETEC, no entanto, em associação com o extrato, foi-se necessária uma concentração de 50,79 $\mu \mathrm{g} \cdot \mathrm{mL}^{-1}$ na ETEC e EPEC. Com isso, constata-se um efeito de modulação aumentado para o aminoglicosídeo amicacina, pois necessitou-se de uma concentração menor do antibiótico em associação com o extrato, para inibir os sorotipos de E. coli.

No aminoglicosídeo gentamicina não houve 
sinergismo e nem antagonismo frente ao sorotipo EPEC, obtendo nas duas situações a mesma concentração de $32 \mu \mathrm{g} \cdot \mathrm{mL}^{-1}$, entretanto no sorotipo ETEC apresentou-se efeito sinérgico, pois o antibiótico isolado foi-se necessário uma concentração de $32 \mu \mathrm{g} \cdot \mathrm{mL}^{-1}$ porém, associado ao extrato, foi-se necessário uma concentração de $12,70 \mu \mathrm{g} \cdot \mathrm{mL}^{-1}$, sendo menor no combate a ETEC.

O efeito do extrato com clindamicina, antibiótico da classe das lincosamidas é mostrado no gráfico 1 e 2. Pode-se evidenciar que não houve sinergismo e nem antagonismo da clindamicina frente as cepas da E. coli.

O presente estudo mostrou a CIM e a modulação do extrato da planta A. satureioides. Segundo Seelig e Grazziotin (2014), os resultados podem ter interferência de fatores naturais, como radiação solar, raios UV, períodos de seca ou chuva, ausência de nutrientes, estação do ano que influenciam na produção de metabólitos secundários pela planta, e estes fatores, aliados à metodologia empregada, influenciam nos resultados obtidos nos ensaios microbianos. $\mathrm{O}$ resultado obtido pode ter relação com a produção do metabólito secundário pela planta, mediante a condição que ela se apresentava.

A associação de antimicrobianos, em casos particulares, com a finalidade de se alcançar uma ação sinérgica ou um aumento do potencial de ação dos mesmos se apresenta como outra alternativa de tratamento. Portanto, fez se necessário a análise da modulação do extrato, frente aos antimicrobianos da classe dos aminoglicosídeos e da classe das lincosamidas (Coutinho et al., 2015).

Os mecanismos pelos quais os extratos podem inibir o crescimento de micro-organismos são variados, e podem ser explicados em parte devido à natureza hidrofóbica de alguns componentes (Dutra et al., 2016). Esses componentes podem interagir com a dupla camada lipídica da membrana celular e afetam a cadeia respiratória e a produção de energia ou, até mesmo, tornam as células mais permeáveis a antibióticos, levando à interrupção da atividade celular (Tintino et al., 2013).

Entretanto, Kadarian et al. (2014) afirma que a alta concentração de flavonoides presentes na $A$. Satureioides tem sido responsável pela atividade biológica relatada pela planta medicinal (Sperotto, 2010). Segundo Polydoro et al. (2004) a composição química da Marcela tem indicado quercitina,3O-metilquercentin e luteolina como os principais flavonoides constituintes. Do mesmo modo, Avancini et al. (2016) afirma que a $A$. satureioides demonstrou a presença de compostos fenólicos, taninos, taninos condensados e de flavonóides tais compostos são comumente encontrados nesta planta e são atribuídos a estas classes de compostos as atividades biológicas da planta.
As doenças do trato gastrointestinal são induzidas por estresse oxidativo e a superprodução de espécies reativas de oxigênio (ROS) se acumulam por condições anormais causando doenças. No entanto, estudos prévios da A. Satureioides demostram que a presença de flavonoides, inibe a peroxidação lipídica por eliminação de ROS, o que provavelmente justifica seu uso popular em doenças gastrointestinais (Moresco et al., 2017).

Both (2014) afirma que o estudo in vitro realizado com a planta medicinal $A$. satureioides comprovou que esta espécie detém atividade bacteriostática e bactericida contra amostras de bactérias gram-positivas padronizadas. Uma das hipóteses é que a planta possua uma maior atividade inibitória para bactérias gram-positivas.

Do mesmo modo Mota (2008) relata que, a $A$. satureioides mostra-se completamente ineficazes frente a algumas bactérias gram-negativas, a exemplo da $E$. coli, o qual mostrou que as formas de extração por infusão e decocção foram completamente ineficazes às bactérias gram-negativas.

Segundo Cruz et al. (2016) estudos relacionados com atividade antibacteriana de plantas medicinais e o sinergismo com fármacos, está sendo frequente, já que essa associação pode servir como auxílio a uma nova estratégia de tratamento. Pois os extratos podem atuar alterando a atividade antibacteriana e, consequentemente, essa modificação acarreta a diminuição da concentração necessária para a inibição do crescimento do microrganismo.

Investigações de alternativas com ação antimicrobiana, poderá abrir caminhos para o desenvolvimento de novos fármacos no combate a resistência bacteriana (Guimarães et al., 2010).

\section{CONCLUSÕES}

A partir da análise dos dados, observou-se que o extrato alcoólico das flores da $A$. satureioides não apresentou atividade antimicrobiana significativa de forma isolada. No entanto, em associação com os aminoglicosídeos apresentou efeitos sinérgicos frente às linhagens testadas, sugerindo-se que a associação dos antibióticos aumenta o seu potencial antimicrobiano.

Nesse contexto, pesquisas posteriores serão importantes para maior certificação de que essa seja uma via alternativa no tratamento de infecções gastrointestinais.

\section{REFERÊNCIAS BIBLIOGRÁFICAS}

Anyanwu MU, Okoye RC. Antimicrobial activity of Nigerian medicinal plants. Journal of intercultural ethnopharmacology, v. 6, n. 2, p. 240, 2017.

https://doi.org/10.5455/jice.20170106073231 
Avancini CAM, Campos FL, Valente P, Ethur EM. Disinfectant activity of the crude hydroalcoholic extract of Achyrocline satureioides (Asteraceae) on Candida spp. isolated in problem-situations of bovine mastitis. Acta Veterinaria Brasilica, v. 10, n. 4, p. 327-333, 2016. https://doi.org/10.21708/avb.2016.10.4.6051

Both JMC. Atividade antibacteriana de desinfetantes convencionais e de extrações de Achyrocline satureioides (Lam.) DC.(Asteraceae)("macela") sobre Staphylococcus aureus meticilina resistentes (MRSA). 2014. 77p. Tese (Doutorado em Ciências Veterinárias) - Universidade Federal do Rio Grande do Sul, Porto Alegre.

Brasil. Ministério da Saúde. Doenças transmitidas por alimentos. Brasília, 2017. Disponível em: <http://portalms.saude.gov.br/saude-de-a-z/doencas-transmitidas-poralimentos> acessado em: 17 agosto de 2018 .

Brasil. Sociedade Brasileira de Pediatria. Diarreia aguda: Diagnóstico e tratamento. Brasília, 2017. Disponível em:<http://www.sbp.com.br/filead$\mathrm{min} /$ user_upload/2017/03/Guia-Pratico-DiarreiaAguda.p $\overline{d f}>$ acessado em: 17 agosto de 2018.

Cariddi LN, Sabini MC, Escobar FM, Bacchetti R, Montironi I, Merckis C, Sabini LI. In Vitro and In Vivo Cytogenotoxic Effects of Hot Aqueous Extract of Achyrocline satureioides (Lam.) DC. BioMed research international, v. 2015, 2015. https://doi.org/10.1155/2015/270973

Coutinho HD, Brito SM, Leite NF, Vandesme VC, Oliveira MT, Martins GM, do Socorro Costa M. Avaliação comparativa da modulação de antibióticos, frente às cepas bacterianas de Escherichia coli, Staphylococcus aureus. Revista Ciencias de la Salud, v. 13, n. 3, p. 345-354, 2015.

Cruz AJ, Brito IP, Sobral MA, Sousa AT, Alves EF, Andreza RDS, Macêdo RO. Avaliação da atividade antibacteriana e moduladora dos extratos metanólico e hexânico da folha de Allium cepa. Ciencias de la salud, v. 14, n. 2, p. 191-200, 2016.

Dutra FSG, de Almeida Carlos L, da Motta OV, Vianna AP, Pereira SMF. Atividade antimicrobiana de extratos vegetais frente à bactérias de importância médica. Biológicas \& Saúde, v. 6, n. 20, 2016.

Farfán-García AE, Ariza-Rojas SC, Vargas-Cárdenas FA, Vargas-Remolina LV. Mecanismos de virulencia de Escherichia coli enteropatógena. Revista chilena de infectología, v. 33, n. 4, p. 438-450, 2016.

http://dx.doi.org/10.4067/S0716-10182016000400009

Gomes TA, Elias WP, Scaletsky IC, Guth BE, Rodrigues JF, Piazza RM, Martinez MB. Diarrheagenic Escherichia coli. Brazilian journal of microbiology, v. 47, p. 3-30, 2016. https://doi.org/10.1016/j.bjm.2016.10.015

Gomes LO, de Matos HJ, de Moraes Silva MC, Loureiro ECB, Macarenhas JDAP, Gabbay YB, da Cruz Rocha DC. Aspectos epidemiológicos das enteroinfecções bacterianas em menores de 5 anos de idade em Rio Branco, estado do Acre, Brasil. Rev Pan-Amaz Saude, v. 8, n. 4, p. 35-43, 2017.

Guimarães DO, Momesso LDS, Pupo MT. Antibióticos: importância terapêutica e perspectivas para a descoberta e desenvolvimento de novos agentes. Química Nova, v. 33, n. 3, p. 667-679, 2010.
Kadarian C, Broussalis AM, Miño J, Lopez P, Gorzalczany S, Ferraro G, Acevedo C. Hepatoprotective activity of Achyrocline satureioides (Lam) DC. Pharmacological Research, v. 45, n. 1, p. 57-61, 2002. https://doi.org/10.1006/phrs.2001.0904

Moresco KS, Silveira AK, Zeidán-Chuliá F, Correa APF, Oliveria RR, Borges AG, Vale MGR. Effects of Achyrocline satureioides inflorescence extracts against pathogenic intestinal bacteria: chemical characterization, in vitro tests, and in vivo evaluation. Evidence-Based Complementary and Alternative Medicine, v. 2017, 2017. https://doi.org/10.1155/2017/4874865

Mota FM. Atividade antibacteriana "in vitro" de inflorescêcias de Achyrocline satureioides (LAM.) DC- Asteraceae- ("macela", "marcela") como fator de proteção em zoonoses. 2008. 91p. Dissertação (Mestrado em Ciências Veterinárias) - Faculdade de Veterinária, Universidade federal do Rio Grande do Sul, Porto Alegre.

National committee for clinical laboratory standards. M7-a6 Metodologia dos testes de sensibilidade a agentes antimicrobianos por diluição para bactéria de crescimento aeróbico. v. 23, n. 2, p. 1-48, 2003.

Polydoro M, De Souza KCB, Andrades ME, Da Silva EG, Bonatto F, Heydrich J, Moreira JCF. Antioxidant, a prooxidant and cytotoxic effects of Achyrocline satureioides extracts. Life sciences, v. 74, n. 23, p. 2815-2826, 2004. https://doi.org/10.1016/j.lfs.2003.09.073

Retta D, Dellacassa E, Villamil J, Suárez SA, Bandoni AL. Marcela, a promising medicinal and aromatic plant from Latin America: a review. Industrial Crops and Products, v. 38, p. 27-38, 2012. https://doi.org/10.1016/j.indcrop.2012.01.006

Sabini MC, Cariddi LN, Escobar FM, Mañas F, Comini L, Iglesias D, Cantero JJ. Potent inhibition of Western Equine Encephalitis 250ntio by a fraction rich in flavonoids and phenolic acids obtained from Achyrocline satureioides. Revista Brasileira de Farmacognosia, v. 26, n. 5, p. 571-578, 2016. https://doi.org/10.1016/j.bjp.2016.05.004

Salgueiro AC, Folmer V, da Rosa HS, Costa MT, Boligon AA, Paula FR, Puntel GO. In vitro and in silico 250ntioxidante and toxicological activities of Achyrocline satureioides. Journal of ethnopharmacology, v. 194, p. 6-14, 2016. https://doi.org/10.1016/j.jep.2016.08.048

Salvat A, Antonnacci L, Fortunato RH, Suárez EY, Godoy HM. Triagem de algumas plantas do norte da Argentina quanto à atividade antimicrobiana. Cartas em microbiologia aplicada, v. 32, n. 5, pág. 293-297, 2001. https://doi.org/10.1046 / j.1472-765x.2001.00923.x

Seelig AP, Grazziotin NA. Avaliação da atividade antibacteriana de extratos aquosos de inflorescências de achyrocline satureioides de três marcas comerciais. Perspectiva, Erechim. v. 38, n.141, p. 89-98, 2014.

Souza CDO, Melo TRB, Melo CDSB, Menezes ÊM, Carvatho ACD, Monteiro LCR. Escherichia coli enteropatogênica: uma categoria diarreiogênica versátil. Revista PanAmazônica de Saúde, v. 7, n. 2, p. 79-91, 2016. https://doi.org/10.5123/S2176-62232016000200010

Sperotto (2010). Atividade antibacteriana in vitro do decocto de Achyrocline satureioides (Lam.) DC-asteracea-("macela"), sobre bactérias isoladas de mastite bovina. 2010. 
55p. Dissertação (Mestrado em Ciências Veterinárias) Universidade Federal do Rio Grande do Sul, Porto Alegre.

Tintino SR, da Cunha FAB, dos Santos KKA, de Melo Guedes GM, Souza CES, Matias EFF, Coutinho HDM. Atividade moduladora de extratos etanólico e hexânico de raiz de Costus cf. arabicus sobre drogas antimicrobianas. Revista Brasileira de Biociências, v. 11, n. 2, 2013.

Zhou Y, Zhu X, Hou H, Lu Y, Yu J, Mao L, Sun Z. Characteristics of diarrheagenic Escherichia coli among children under 5 years of age with acute diarrhea: a hospital based study. BMC infectious diseases, v. 18, n. 1, p. 63, 2018. https://doi.org/10.1186/s12879-017-2936-1 